DEMONSTRATIO MATHEMATICA

$\begin{array}{lll}\text { Vol. XXVI } & \text { No 3-4 } & 1993\end{array}$

Nguyen Cang, Nguyen Kim Tan

\title{
THEORÈME DE POSITIVITÉ D'UN SYSTÈME DISCRET EN CAS NON COMPLĖTEMENT CONTRÔLABLE ET SON APPLICATION
}

\section{Introduction}

Considérons le système suivant

(1) $x_{k+1}=A x_{k}+B u_{k}, k=0,1, \ldots$

(2) $\eta\left(0, k_{1}\right)=\left[x_{k}^{*} J x_{k}\right]_{0}^{k_{1}+1}+\sum_{k=0}^{k_{1}}\left[u_{k}^{*} K u_{k}+x_{k}^{*} L u_{k}+u_{k}^{*} L^{*} x_{k}+x_{k}^{*} M x_{k}\right]$

dans lequel $\eta$ est appelé "fonction de coût", $u_{k}$ fonction vectorielle de commande à $m$ composantes au moment $k, x_{k}$ vecteur à $n$ composantes qui décrit l'état du système au moment $k$, et les matrices constantes: $A, J, M$ à $n$ lignes et $n$ colonnes, $B, L$ à $n$ lignes et $m$ colonnes, $K$ à $m$ lignes, $m$ colonnes. Les matrices $K, J, M$ sont supposées hermitiennes (c'est-à-dire $K=K^{*}, J=J^{*}, M=M^{*}$; le signe * veut dire transposer et prendre le conjugué).

Le couple $(A, B)$ n'étant pas complèment contrôlable (c.c), d'après la proposition 3 de [1] (p. 342), il existe une matrice non singulière $R$ telle que

$$
\tilde{A}=R A R^{-1} \quad \text { et } \quad \tilde{B}=R B, \quad \tilde{A}=\left(\begin{array}{cc}
A_{11} & A_{12} \\
0 & A_{22}
\end{array}\right), \quad \tilde{B}=\left(\begin{array}{c}
B_{1} \\
0
\end{array}\right)
$$

avec les matrices constantes $A_{11}$ à $k$ lignes et $k$ colonnes $(k<n$, eventuellement $k=0), A_{12}$ à $k$ lignes et $n-k$ colonnes, $A_{22}$ à $n-k$ lignes et $n-k$ colonnes; $\left(A_{11}, B_{1}\right)$ étant c.c. Si l'on applique la transformation $\widetilde{x}=R x$ et si l'on met

$$
\tilde{x}=\left(\begin{array}{c}
y_{k} \\
z_{k}
\end{array}\right), \quad J=\left(\begin{array}{ll}
J_{11} & J_{12} \\
J_{12}^{*} & J_{22}
\end{array}\right), \quad M=\left(\begin{array}{ll}
M_{11} & M_{12} \\
M_{12}^{*} & M_{22}
\end{array}\right), \quad L=\left(\begin{array}{l}
L_{1} \\
L_{2}
\end{array}\right),
$$


alors le système (1) se transforme en

$$
\left\{\begin{array}{l}
y_{k+1}=A_{11} y_{k}+A_{12} z_{k}+B_{1} u_{k} \\
z_{k+1}=A_{22} z_{k}
\end{array}\right.
$$

Remarquons qu'on peut reécrire (2) sous la forme plus compacte

$$
\eta\left(0, k_{1}\right)=\left[x_{k}^{*} J x_{k}\right]_{0}^{k_{1}+1}+\sum_{k=0}^{k_{1}} s_{k}^{*} D s_{k}
$$

avec

$$
s_{k}=\left(\begin{array}{c}
x_{k} \\
u_{k}
\end{array}\right), \quad D=\left(\begin{array}{cc}
K & L^{*} \\
L & M
\end{array}\right) .
$$

On voit aisément que $D=D^{*}$.

V. M. Popov [1] a énoncé le théorème de positivité du système (1), (2) en cas du couple $(A, B)$ étant c.c. Dans ce qui suit nous établissons un tel théorème pour $(A, B)$ n'étant pas c.c.

2. Théorème de positivité pour $(A, B)$ n'étant pas c.c.

Nous rappelons, suivant [1], les définitions suivantes.

DÉfinition 2.1. Le système (1), (2) est appelé positif, si $\eta\left(0, k_{1}\right)$ peut être ramené à la forme suivante

$$
\eta\left(0, k_{1}\right)=[\alpha(x)]_{0}^{k_{1}+1}+\sum_{k=0}^{k_{1}} \beta\left(x_{k}, u_{k}\right) \text { avec } \beta\left(x_{k}, u_{k}\right) \geq 0 .
$$

Définition 2.2. La matrice caractéristique $H(\lambda, \sigma)$ et le polynôme caractéristique $\Pi(\lambda, \sigma)$ sont définis par les formules suivantes

$$
\left\{\begin{aligned}
H(\lambda, \sigma)= & K+L_{1}^{*}\left(\sigma E_{1}-A_{11}\right)^{-1} B_{1}+B_{1}^{*}\left(\lambda E_{1}-A_{11}^{*}\right)^{-1} L_{1} \\
& +B_{1}^{*}\left(\lambda E_{1}-A_{11}^{*}\right)^{-1}\left[M_{11}+(\lambda \sigma-1) J_{11}\right]\left(\sigma E_{1}-A_{11}\right)^{-1} B_{1} \\
\Pi(\lambda, \sigma)= & \operatorname{det}\left(\sigma E_{1}-A_{11}\right) \operatorname{det} H(\lambda, \sigma) \operatorname{det}\left(\lambda E_{1}-A_{11}^{*}\right)
\end{aligned}\right.
$$

dans lesquels $\lambda, \sigma$ sont des variables complexes et $E_{1}$ une matrice unité dont la dimension est celle de $A_{11}$.

Supposons que le système (1), (2) vérifient les conditions suivantes

$\left(\mathrm{C}_{1}\right) \Pi\left(\sigma^{-1}, \sigma\right) \not \equiv 0$

$\left(\mathrm{C}_{2}\right) A_{22}$ est une matrice invertible;

$\left(\mathrm{C}_{3}\right)$ les polynômes $\operatorname{det}\left(\sigma E_{2}-\left(A_{22}^{-1}\right)^{*}\right)$ et $\Pi\left(\sigma^{-1}, \sigma\right)$ n'ont pas de racines communes dans le disque $|\sigma| \leq 1$, où $E_{2}$ est une matrice unité dont la dimension est celle de $\boldsymbol{A}_{22}$;

$\left(C_{4}\right)$. les racines $\lambda_{j}$, du polynôme $\operatorname{det}\left(\lambda E_{22}-A_{22}\right)$ vérifient la relation $\bar{\lambda}_{i} \lambda_{j}, \neq 1$ avec $i, j \in\left\{1,2, \ldots, \operatorname{dim} A_{22}\right\}$. 
THÉORÈme 2.1. Si les conditions $\left(\mathrm{C}_{1}\right)-\left(\mathrm{C}_{4}\right)$ sont vérifiées, les propositions suivantes sont équivalentes:

1) Le système (1), (2) est positif.

2) $H\left(\sigma^{-1}, \sigma\right) \geq 0$ (semi-définie positive) avec tout $\sigma$ vérifiant $\operatorname{det}\left(\sigma E_{1}-\right.$ $\left.A_{11}\right) \neq 0$ et $|\sigma|=1$.

3) Il existe au moins un polynôme $\psi(\sigma)$ qui vérifie soit $\psi(\sigma) \equiv 0$ soit $\psi(\sigma) \neq 0$ de manière que

(3.a) $\Pi\left(\sigma^{-1}, \sigma\right)=\bar{\psi}\left(\sigma^{-1}\right) . \psi(\sigma), \psi(\sigma) \neq 0$ implique $\psi(0) \neq 0$ avec $\bar{\psi}\left(\sigma^{-1}\right)=\overline{\psi\left(\bar{\sigma}^{-1}\right)}$

(3.b) il existe une matrice hermitienne $N$ et des matrices $V, W$ telles que $H\left(\sigma^{-1}, \sigma\right)={\overline{F_{0}}}^{T}\left(\sigma^{-1}\right) . F_{0}(\sigma)$ (notons que $\overline{F_{0}}\left(\sigma^{-1}\right)=\overline{F_{0}\left(\bar{\sigma}^{-1}\right)}$ );

(3.c) $F_{0}(\sigma)=V+W^{*}(\sigma E-A)^{-1} B$;

(3.d) $\operatorname{det} F_{0}(\sigma)=\sqrt{\nu} \frac{\psi(\sigma)}{\operatorname{det}\left(\sigma E_{1}-A_{11}\right)}, \nu \in \mathcal{R}^{+}$, $\sqrt{\nu}$ étant le coefficient de factorisation;

(3.e) $\left(\begin{array}{cc}K & L^{*} \\ L & M\end{array}\right)+\left(\begin{array}{cc}B^{*} N B & B^{*} N A \\ A^{*} N B & A^{*} N A-N\end{array}\right)=\left(\begin{array}{cc}V^{*} V & V^{*} W^{*} \\ W V & W W^{*}\end{array}\right)$;

(3.f) $\eta\left(0, k_{1}\right)$ peut être reécrit sous la forme $\eta\left(0, k_{1}\right)=\left[x_{k}^{*}(J-N) x_{k}\right]_{0}^{k_{1}+1}$ $+\sum_{k=0}^{k_{1}}\left|V u_{k}+W^{*} x_{k}\right|^{2}$

4) Il existe une matrice hermitienne $N$ telle que

$$
\tilde{D}=\left(\begin{array}{cc}
K+B^{*} N B & \left(L+A^{*} N B\right)^{*} \\
L+A^{*} N B & M+A^{*} N A-N
\end{array}\right) \geq 0 .
$$

La démonstration de ce théorème est analogue à celle de [2].

\section{Application}

Puisque $(A, B)$ n'est pas c.c., reprenons le système (3), où $\left(A_{11}, B_{1}\right)$ est c.c. et

$\eta(0, \infty)=\lim _{k \rightarrow \infty} \eta(0, k), \quad \eta(0, k)$ étant donné par $(2)$.

DÉfinition 3.1. $\left(y_{k}, z_{k}\right)_{k \in \mathcal{N}}$ est appelé solution du système (3), s'il vérifie les conditions suivantes

(i) $\left(y_{k}, z_{k}\right)_{k \in \mathcal{N}}$ vérifie le système (3),

(ii) $\lim _{k \rightarrow \infty} y_{k}=0, \lim _{k \rightarrow \infty} z_{k}=0$,

(iii) $\eta(0, \infty)<+\infty$.

ThÉORÈme 3.1. Supposons que les conditions suivantes soient vérifiées

1) la matrice $K+B^{*} N B$ est invertible, 
2) $\Pi\left(\sigma^{-1}, \sigma\right) \neq 0, \forall \sigma \in \mathcal{C}:|\sigma|=1$,

3) toute valeur propre $\lambda$ de $A_{22}$ vérifie $0<|\lambda|<1$.

En outre, si le système (3) vérifie l'une des conditions suivantes:

(4.i) $D=\left(\begin{array}{ll}K & L^{*} \\ L & M\end{array}\right) \geq 0$,

(4.ii) la matrice caractéristique $H\left(\sigma^{-1}, \sigma\right)$ est semi-définie positive, i.e.

$$
H\left(\sigma^{-1}, \sigma\right) \geq 0, \quad \forall \sigma \in \mathcal{C}:|\sigma|=1, \quad \text { et } \operatorname{det}\left(\sigma E_{1}-A_{11}\right) \neq 0,
$$

alors le système (3) admet la fonction de commande optimale unique qui minimise la fonction de coût $\eta(0, \infty)$.

Preuve. Remarquons que les conditions $\left(\mathrm{C}_{2}\right)-\left(\mathrm{C}_{4}\right)$ serons vérifiées si

$\left(\mathrm{C}_{5}\right)$ toute valeur propre de la matrice $A_{22}$ est dans le disque $\{\lambda \in \mathcal{C}$ : $0<|\lambda|<1\}$.

En effet, la matrice $\boldsymbol{A}_{22}$ n'a pas de valeur propre nulle, $\operatorname{donc} \operatorname{det} \boldsymbol{A}_{22} \neq 0$, d'où $A_{22}$ est invertible, et l'on a $\left(\mathrm{C}_{2}\right)$. Supposons $\operatorname{det}\left(\sigma E_{2}-\left(A_{22}^{-1}\right)^{*}\right)=0$, alors $\bar{\sigma}^{-1}$ est aussi une valeur propres de $A_{22}$, d'où

$$
|\sigma|=\left|\frac{1}{\bar{\sigma}^{-1}}\right|>1
$$

et on trouve $\left(\mathrm{C}_{3}\right)$. Enfin pour tous valeurs propres $\lambda_{i}, \lambda_{j}$ de la matrice $A_{22}$ on a $\left|\lambda_{i} \lambda_{j}\right|<1$, d'où $\left(\mathrm{C}_{4}\right)$ est vérifiée.

Pour démontrer le Théorème 3.1, remarquons que la condition 3) n'est que $\left(\mathrm{C}_{5}\right)$. Ainsi la contrôlabilité du couple $\left(A_{11}, B_{1}\right)$ et les conditions 2) et 3) vérifient toutes les hypothèses du Théorème 2.1. Fixons $k_{1} \in \mathcal{N}$. La condition 4) indique que le système (1), (2), et aussi (3), est positif. La condition (4.ii) est identique à 2 ) du Théorème 2.1. D'où les relations (3.a)(3.f) sont vérifiées. Nous allons démontrer que le polynôme $\psi(\sigma)$ peut être tellement choisi que le module de chacune de ses racines est plus petit que l'unité. En effet, si $\sigma_{0}$ est une racine du polynôme caractéristique $\Pi\left(\sigma^{-1}, \sigma\right)$, alors $\bar{\sigma}_{0}^{-1}$ l'est aussi, puisqu'on a $\Pi\left(\bar{\sigma}, \bar{\sigma}^{-1}\right)=\overline{\Pi\left(\sigma^{-1}, \sigma\right)}$. Or $\left|\sigma_{0}\right| \cdot\left|\sigma_{0}^{-1}\right|=1$, nous pouvons donc, sans perdre de généralité, prendre $\left|\sigma_{0}\right| \leq 1$. Suivant la condition 3) du Théorème 2.1 , on peut tellement choisir $\sigma_{0}$ que $\psi\left(\sigma_{0}\right)=$ $0 \Rightarrow\left|\sigma_{0}\right| \leq 1$. Or, si $\left|\sigma_{0}\right|=1$, alors $\Pi\left(\sigma_{0}^{-1}, \sigma_{0}\right)=0$, ce qui est contraire à la condition 2) du Théorème 3.1. Donc $\psi(\sigma)=0 \Rightarrow|\sigma|<1$.

Nous prouvons ensuite que la matrice $V$ est invertible. Or, en tenant compte de la condition 3) du Théorème 2.1, nous avons $K+B^{*} N B=V^{*} V$. D'où l'invertibilité de la matrice $V$ se déduit aisément de l'hypothèse 1) du Théorème 3.1. 
Pour la fonction de commande $\widehat{u}_{k}=-V^{-1} W^{*} \widehat{x}_{k}$ nous avons

$$
\begin{aligned}
\widehat{x}_{k+1} & =A \widehat{x}_{k}+B \widehat{u}_{k}=\left(A-B V^{-1} W^{*}\right) \widehat{x}_{k} \\
& =\left(\begin{array}{cc}
A_{11}-B_{1} V^{-1} W_{1}^{*} & A_{12}-B_{1} V^{-1} W_{2}^{*} \\
0 & A_{22}
\end{array}\right) \cdot\left(\begin{array}{l}
\widehat{y}_{k} \\
\widehat{z}_{k}
\end{array}\right) .
\end{aligned}
$$

Vu Théorème 2.1, on peut écrire

(5) $\operatorname{det}\left(\sigma E-\left(A-B V^{-1} W^{*}\right)\right)$

$$
\begin{aligned}
& =\operatorname{det}\left(\sigma E_{1}-\left(A_{11}-B_{1} V^{-1} W_{1}^{*}\right)\right) \cdot \operatorname{det}\left(\sigma E_{2}-A_{22}\right) \\
& =V^{-1}\left(V+W_{1}^{*}\left(\sigma E_{1}-A_{11}\right)^{-1} B_{1}\right) \cdot \operatorname{det}\left(\sigma E_{1}-A_{11}\right) \cdot \operatorname{det}\left(\sigma E_{2}-A_{22}\right) \\
& =V^{-1} \sqrt{\nu} \psi(\sigma) \operatorname{det}\left(\sigma E_{2}-A_{22}\right)^{1)} .
\end{aligned}
$$

Les polynômes $\psi(\sigma)$ et $\operatorname{det}\left(\sigma E_{2}-A_{22}\right)$ n'ont pas de racines $\sigma$ avec $|\sigma| \geq 1$ donc la matrice $\left(A-B V^{-1} W^{*}\right)$ est stable, d'où $\lim _{k \rightarrow \infty} x_{k}=0$.

Ayant la condition initiale $x_{0}$ quelconque, posons $\widehat{x}_{0}=x_{0}$. D'après (3) et en tenant compte de $\widehat{u}=-V^{-1} W^{*} \widehat{x}_{k}$, on a

$$
\begin{aligned}
\hat{\eta}(0, \infty)= & \lim _{k_{1} \rightarrow \infty} \hat{\eta}\left(0, k_{1}\right) \\
= & \lim _{k_{1} \rightarrow \infty}\left\{\left[\widehat{x}_{k_{1}+1}^{*}(J-N) \widehat{x}_{k_{1}+1}-\widehat{x}_{0}^{*}(J-N) \widehat{x}_{0}\right]\right. \\
& \left.+\sum_{k=0}^{k_{1}}\left|V \widehat{u}_{k}+W^{*} \widehat{x}_{k}\right|^{2}\right\} \\
= & \lim _{k_{1} \rightarrow \infty}\left[\widehat{x}_{k_{1}+1}^{*}(J-N) \widehat{x}_{k_{1}+1}-\widehat{x}_{0}^{*}(J-N) \widehat{x}_{0}\right] \\
= & \widehat{x}_{0}^{*}(J-N) \widehat{x}_{0}=x_{0}^{*}(J-N) x_{0} .
\end{aligned}
$$

Ainsi, $\left(\widehat{x}_{k}, \widehat{u}_{k}\right)_{k \in \mathcal{N}}$ est une solution de $(3)$ avec $\hat{\eta}(0, \infty)=x_{0}^{*}(N-J) x_{0}<$ $+\infty$. Soit maintenant $\left(x_{k}, u_{k}\right)_{k \in \mathcal{N}}$ une solution de (3). D'après (3.f), on à

$$
\eta\left(0, k_{1}\right)=\left[x_{k}^{*}(J-N) x_{k}\right]_{0}^{k_{1}+1}+\sum_{k=0}^{k_{1}}\left|V u_{k}+W^{*} x_{k}\right|^{2},
$$

d'où il vient

$$
\eta(0, \infty)=\lim _{k_{1} \rightarrow \infty} \eta\left(0, k_{1}\right)=x_{0}^{*}(N-J) x_{0}+\sum_{k=0}^{\infty}\left|V u_{k}+W^{*} x_{k}\right|^{2} \geq x_{0}^{*}(N-J) x_{0} .
$$

1) Dans l'analyse matricielle nous avons la proposition

$$
\operatorname{det}\left(\begin{array}{cc}
A & C \\
D^{*} & B
\end{array}\right)=\operatorname{det} A \cdot \operatorname{det}\left(B-D^{*} A^{-1} C\right)
$$

pour $A$ non singulière. 
Donc $\eta(0, \infty) \geq \widehat{\eta}(0, \infty)$. L'égalité aura lieu pour $u_{k}=-V^{-1} W^{*} x_{k}, k \in \mathcal{N}$. Enfin, on conclut que $\left(\widehat{x}_{k}, \widehat{u}_{k}\right)_{k \in \mathcal{N}}$ est la solution optimale du système (3). Théorème 3.1 est donc démontré.

\section{Références}

[1] V. M. Popov, Hyperstability of control systems, Springer Verlag, 1973.

[2] V. M. Popov, Incompletely controllable positive systems and application to optimisation and stability of automatic control systems, Rev. Roum. Sci. Tech. Electrotechn. et Energ. 12 (1967).

[3] W. Rudin. Real and complex analysis, New York, 1974.

[4] P. Lancaster, Theory of matrices, New York, 1969.

[5] N. Cang and N.K. T an, Communications mathématiques 1990:

1. Optimisation d'une class de systèmes discrets.

2. Positivité et optimisation d'une classe de systèmes discrets.

3. Optimisation et la résolution des équations de Lourié.

DÉPARTEMENT DE MATHÉMATIQUES

UNIVERSITE D'HO CHI MINH VILLE

227 NGUYEN VAN CU, Q.5

HO CHI MINH VILLE - VIET NAM

Received July 17,1991. 